\title{
Comparison of patients undergoing switching versus augmentation of antipsychotic medications during treatment for schizophrenia
}

This article was published in the following Dove Press journal:

Neuropsychiatric Disease and Treatment

14 March 2012

Number of times this article has been viewed

\section{Haya Ascher-Svanum \\ Alan JM Brnabic \\ Anthony H Lawson \\ Bruce J Kinon \\ Virginia L Stauffer \\ Peter D Feldman \\ Katarina Kelin}

Lilly Research Laboratories, Eli Lilly and Company, Lilly Corporate Center, Indianapolis, Indiana, USA
Correspondence: Haya Ascher-Svanum Lilly Research Laboratories,

Eli Lilly and Company, Lilly Corporate

Center, Indianapolis, IN 46285, USA

$\mathrm{Tel}+\mathrm{I} 3172778713$

Fax + I 3172770490

Email haya@lilly.com

\begin{abstract}
It is often difficult to determine whether a patient may best benefit by augmenting their current medication or switching them to another. This post-hoc analysis compares patients' clinical and functional profiles at the time their antipsychotic medications were either switched or augmented. Adult outpatients receiving oral antipsychotic treatment for schizophrenia were assessed during a 12-month international observational study. Clinical and functional measures were assessed at the time of first treatment switch/augmentation ( $0-14$ days prior) and compared between Switched and Augmented patient groups. Due to low numbers of patients providing such data, interpretations are based on effect sizes. Data at the time of change were available for 87 patients: 53 Switched and 34 Augmented. Inadequate response was the primary reason for treatment change in both groups, whereas lack of adherence was more prevalent in the Switched group (26.4\% vs $8.8 \%)$. Changes in clinical severity from study initiation to medication change were similar, as indicated by Clinical Global Impressions-Severity scores. However, physical and mental component scores of the 12-item Short-Form Health Survey improved in the Augmented group, but worsened in the Switched group. These findings suggest that the patient's worsening or lack of meaningful improvement prompts clinicians to switch antipsychotic medications, whereas when patients show some improvement, clinicians may be more likely to try bolstering the improvements through augmentation. Current findings are consistent with physicians' stated reasons for switching versus augmenting antipsychotics in the treatment of schizophrenia. Confirmation of these findings requires further research.
\end{abstract}

Keywords: augmentation, drug substitution, polypharmacy, schizophrenia, switching

\section{Introduction}

Treatment optimization for patients with schizophrenia remains a challenge, considering that approximately one-third of patients are resistant to a particular treatment, ${ }^{1}$ and as many as half of all patients in a given year will have their antipsychotic medications switched. ${ }^{2}$ Although current treatment guidelines ${ }^{3-5}$ recommend monotherapy as a first-line strategy, switching to another medication is recommended for patients experiencing no relief of symptoms. Furthermore, if multiple attempts at monotherapy fail, augmentation - the use of a combination of agents - is suggested. In usual care, antipsychotic medication-use patterns often do not match treatment guidelines, and the drivers of antipsychotic polypharmacy in the treatment of schizophrenia are not well understood. Surveys indicate that physicians choose medication augmentation to bolster a patient's partial or suboptimal improvements, particularly of positive symptoms, whereas lack of response or clinical worsening leads to the decision to switch the patient to another antipsychotic medication. ${ }^{6-8}$ To the best of our knowledge, there are 
no publications of "real world" studies in which the clinical or functional profiles of patients with schizophrenia have been assessed at the time their antipsychotic medications were switched or augmented. Moreover, there are no prospective studies in usual care in which treating physicians were queried about their reasons for switching or augmenting medications in the treatment of patients with schizophrenia.

The opportunity to expand our understanding of what drives the decision to switch or augment a patient's antipsychotic medication has become available recently in a 12-month, prospective, observational study of schizophrenia in usual practice settings. ${ }^{9}$ This study assessed the clinical and functional status of patients at the time of oral antipsychotic medication switch/augmentation, as well as physicians' reasons for making these changes, at the time the treatment changes took place. The objectives of the current exploratory, post-hoc analysis, which was based on data from that study, were twofold. The primary objective was to compare the clinical and functional profiles of patients undergoing treatment switching or augmentation by examining (A) scores at study entry, (B) scores at the time of their first switch/augmentation following study initiation, and (C) change scores from study entry to the time of their first switch/augmentation. In addition, this study's secondary objective was to compare reasons for the switching versus augmentation, as reported by the patients' treating physicians at the time these changes were made in patients' medication regimens.

\section{Methods}

We used data from an international, 12-month, prospective, observational, noninterventional study (study F1DAY-B033), ${ }^{9}$ conducted in Australia, Mexico, Romania, and Taiwan, which examined time to all-cause treatment discontinuation in adult outpatients diagnosed with schizophrenia (Diagnostic and Statistical Manual of Mental Disorders, fourth edition, or fourth edition, text revision). Inclusion criteria included a need to switch from ongoing antipsychotic treatment to another antipsychotic due to a risk of medication nonadherence per treating clinicians' perceptions and an experience of at least two previous episodes of clinical worsening in the preceding 24 months that required hospitalization or an increased level of care. The time of this initial switch constituted the time of entry to the study. This analysis focuses on those patients who were switched at study entry to an oral antipsychotic. Patients were excluded from the study if they were considered by their clinician to be treatment-resistant.
After procedures and possible side effects were explained to them, all participating patients provided written consent to participate in the study, which was conducted in accordance with the Declaration of Helsinki and all applicable local laws and regulations.

In addition to examination of patient demographics and illness characteristics at study entry, assessments were made of the number of patients who were hospitalized in the 24 months prior to study initiation, and previous antipsychotic use was noted. Physicians' reported reasons for medication switch/augmentation after study initiation were documented, and times to switch/augmentation after study initiation and times to all-cause discontinuation from the study were recorded. Clinical and functional measures were assessed at study entry and at the time of the first treatment switch/augmentation (the most recent observation within the 14 days preceding the change) after study entry. Changes in these measures from study entry to the first switch/ augmentation event after study initiation were calculated. Severity of patients' illness was measured with the Clinical Global Impressions-Severity scale (CGI-S), ${ }^{10}$ with scores ranging from $1=$ "normal/not at all ill" to $7=$ "among the most extremely ill patients." Patients' functioning and wellbeing were measured with the physical and mental component scores of the patient-rated, 12-item Short-Form Health Survey (SF-12), ${ }^{11}$ with scores standardized to a scale of 0 to 100 , where higher scores represent better functioning. Healthrelated quality of life was assessed with the utility index and health state scores of the patient-reported European Quality of Life-5-Dimensions scale (EQ-5D). ${ }^{12}$ Each dimension is rated from 1 = "better health state (no problems)" to $3=$ "worst health state (confined to bed)" and transformed to a single utility index score scaled from $0=$ death to $1=$ perfect health. The health state score is scaled from $0=$ "worst imaginable health state" to $100=$ "best imaginable health state." Patients' attitudes towards the medication intake were assessed with the self-reported 10-item Drug Attitude Inventory (DAI-10), ${ }^{13}$ with a range of -10 to +10 , higher scores reflecting better attitudes. Patients' insight into their illness was measured with the Scale to Assess Unawareness of Mental DisorderAbbreviated version (SUMD-A), ${ }^{14}$ a semistructured, nine-item, open interview with item scores ranging from $1=$ "aware" to 3 = "severely unaware." The SUMD-A total score is computed from the total of all nine items, with a range of 1 to 27 , higher scores indicating more awareness.

Differences at study entry between the Switched and Augmented patient groups were analyzed using descriptive statistics. Time to first switch/augmentation after study 
initiation and time to discontinuation from the study were calculated from the date of study entry until the date of the event. Patient data were censored from the analysis if the patient had completed the study, died, or was lost to follow-up. Results were analyzed using descriptive statistics and, where appropriate, Kaplan-Meier ${ }^{15}$ estimates for the median. Comparison of assessment scores at the time of event (switching/augmentation) and analysis of changes from study entry to event were performed using ANOVA and examination of standardized mean-difference effect sizes (Cohen's d). ${ }^{16}$ However, no adjustment was made because no differences were observed between groups at baseline, the sample sizes were small, and covariate information was missing.

\section{Results}

Study F1D-AY-B033 enrolled a total of 406 patients, of whom 363 patients were switched at study entry to another oral antipsychotic, and 43 were switched at study entry to a depot antipsychotic. Of the 363 patients switched to an oral antipsychotic at study entry, 87 experienced at least one subsequent treatment switch or augmentation and provided data within 14 days prior to that switch/augmentation event (53 Switched, 34 Augmented). Of the other 276 patients, 207 completed the study, 29 discontinued, 36 were lost to follow-up and could not be classified (switched, augmented, stopped, or discontinued), and four stopped their medication during the observation period. Some of the 87 patients included in this analysis did not provide data for all of the measures assessed at the time of their first switch/ augmentation following study entry; thus, sample sizes varied from one measure to another.

Demographic, clinical, and functional characteristics at study entry of the included 87 patients did not differ significantly from the overall patient sample. Their mean age was approximately 37 years, males slightly outnumbered females, and mean CGI-S scores at study entry (Table 1) indicated moderate to marked illness severity. Mean SF-12 scores indicated levels of functioning approximately 0.5 to 1.5 standard deviations below the overall population norm, while EQ-5D scores indicated an average-to-poor health-related quality of life. Scores on the SUMD-A suggested a moderate level of patients' insight into their illness, while DAI-10 scores indicated moderately positive attitudes towards the medications being taken.

Patients' clinical and functional status at the time of first treatment switch/augmentation suggested greater improvement in the Augmented group than the Switched
Table I Measures at study entry for patients whose antipsychotic medication was Switched $(n=53)$ or Augmented $(n=34)$ during the 12-month study period

\begin{tabular}{|c|c|c|}
\hline Measure & $\mathbf{n}$ & Mean (SD) \\
\hline \multicolumn{3}{|l|}{ CGI-S } \\
\hline Switched & 53 & $4.10(1.02)$ \\
\hline Augmented & 34 & $4.30(0.99)$ \\
\hline \multicolumn{3}{|l|}{ SF-12 PCS } \\
\hline Switched & 53 & $46.25(9.89)$ \\
\hline Augmented & 34 & $41.95(9.58)$ \\
\hline \multicolumn{3}{|l|}{ SF-12 MCS } \\
\hline Switched & 53 & $35.27(10.17)$ \\
\hline Augmented & 34 & $37.17(10.80)$ \\
\hline \multicolumn{3}{|l|}{ EQ-5D utility } \\
\hline Switched & 51 & $0.68(0.22)$ \\
\hline Augmented & 31 & $0.64(0.29)$ \\
\hline \multicolumn{3}{|c|}{ EQ-5D Health State } \\
\hline Switched & 53 & $62.15(21.51)$ \\
\hline Augmented & 34 & $53.97(26.24)$ \\
\hline \multicolumn{3}{|l|}{ SUMD-A } \\
\hline Switched & 47 & $|2.8|(4.33)$ \\
\hline Augmented & 30 & $12.27(5.10)$ \\
\hline \multicolumn{3}{|l|}{ DAI- 10} \\
\hline Switched & 51 & $2.82(4.80)$ \\
\hline Augmented & 33 & $3.03(4.48)$ \\
\hline
\end{tabular}

Abbreviations: CGI-S, Clinical Global Impressions-Severity scale; DAI-10, 10-item Drug Attitude Inventory; EQ-5D, 5-dimension European Quality of Life scale; MCS, mental health component score; PCS, physical component score; SF-12, I2-item Short-Form Health Survey; SD, standard deviation; SUMD-A, Scale to Assess Unawareness of Mental Disorder-Abbreviated version.

group (Figure 1). Although CGI-S scores changed very little in either group, both SF-12 and EQ-5D scores showed that the Augmented patient group experienced a slight improvement in mean functioning and health-related quality of life, whereas Switched patients showed no change or a slight worsening. Effect sizes were small to medium, with the exception of the effect size associated with changes in the SF-12 physical component score, which was large (and the only measure associated with a statistically significant improvement, despite the very small sample sizes). The latter also indicated improvement in the Augmented group and a worsening in the Switched group. Drug attitudes, as indicated by DAI-10 scores, were essentially unchanged in the Augmented group but worsened in the Switched group, with a small effect size associated with the difference between patient groups. Similarly, patients' insights into their illness, as measured by the SUMD-A, were essentially unchanged in the Augmented group but worsened in the Switched group, with a medium effect size associated with the difference between patient groups. In lieu of median Kaplan-Meier estimates, the median time to event (switch, augmentation, or stop) was slightly longer in the Switched group 
A

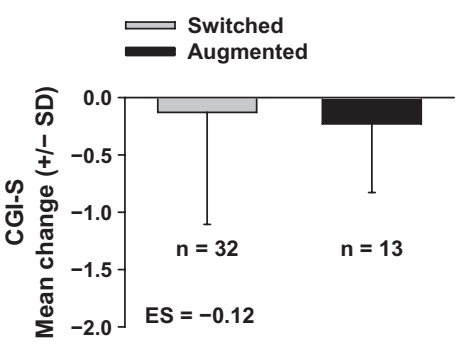

B
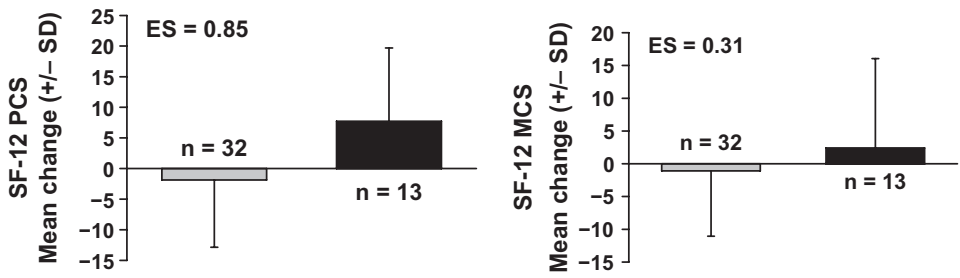

C
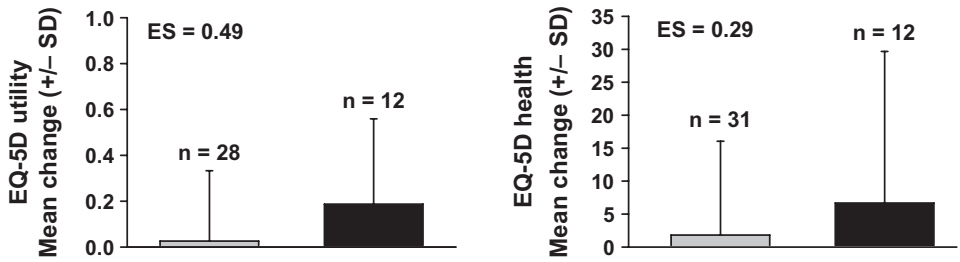

D
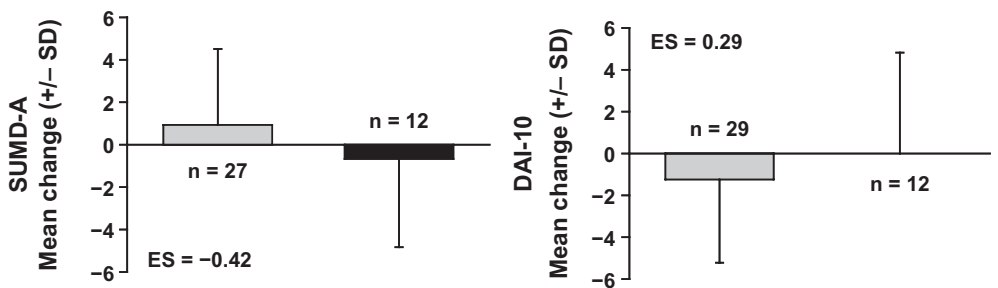

Figure I Effect sizes and changes from study entry to time of first switch or augmentation among patients experiencing a switch to (gray bars), or augmentation with (black bars), another oral antipsychotic following study initiation: (A) illness severity, as measured by the CGI-S, (B) functioning and well-being, as measured by the SF-I 2 physical (left) and mental component score (right), (C) quality of life, as measured by the EQ-5D utility index (left) and health state score (right), and (D) attitude towards medication intake and insight into illness, as measured by the SUMD-A (left) and DAI-I0 (right), respectively.

Notes: $P=0.01$, difference between mean SF-12 PCS change scores for Switched and Augmented groups. All other measures were not statistically significant.

Abbreviations: CGI-S, Clinical Global Impressions-Severity scale; DAI-I0, 10-item Drug Attitude Inventory; EQ-5D, 5-dimension European Quality of Life scale; MCS, mental health component score; ES, effect size for Augmented patient group, relative to Switched patient group, on change scores from study entry to time of first switch/augmentation event; PCS, physical component score; SF-12, I2-item Short-Form Health Survey; SD, standard deviation; SUMD-A, Scale to Assess Unawareness of Mental Disorder-Abbreviated version.

(Switched: median $=5.5$ months, minimum to maximum $=0$ to 12.8 months, $\mathrm{n}=53$; Augmented: median $=3.6$ months, minimum to maximum $=0$ to 10.7 months, $\mathrm{n}=34$ ). The median time to all-cause study discontinuation (dropout) was similar for the two groups (Switched: median 12.2 months, minimum to maximum $=2.1$ to 14.5 months, $\mathrm{n}=53$; Augmented: median $=12.3$ months, minimum to maximum $=3.1$ to 13.9 months, $\mathrm{n}=34$ ).

Reasons physicians' reported for switching or augmenting their patients' medications were generally not appreciably different between the two patient groups, with inadequate response being the leading reason in both groups (Switched: 18 of 53 , or $34.0 \%$; Augmented: 14 of 34 , or $41.2 \%$ ). However, lack of adherence was somewhat more frequently observed in the Switched group, nearly attaining statistical significance (Switched: 14 of 53, or 26.4\%; Augmented: three of 34 , or $8.8 \%$ ). Lack of tolerance was a less common reason for treatment change and was not different between patient groups (Switched: four of 53, or 7.5\%; Augmented: three of 34 , or $8.8 \%$ ). 


\section{Discussion}

This study offers, for the first time, empirical corroboration of previous reports that physicians tend to switch a patient's antipsychotic medication when there is lack of improvement, ${ }^{17,18}$ whereas they opt for augmentation in an attempt to consolidate clinical gains and optimize responses to medication. ${ }^{1,6}$ Patients in this analysis who underwent either a switch or an augmentation of their antipsychotic medication did not differ at the outset of the study. However, patients whose medications were switched after study entry had been worsening during the period leading up to the treatment change on most clinical and functional outcome measures, whereas patients whose medications were augmented had been showing some improvement. Interestingly, the primary reason for the treatment change, as reported by the treating physicians, was essentially the same for the two groups: lack of efficacy. This is consistent with the reasons for switching or augmentation that have been reported in a large systematic review of longitudinal antipsychotic prescriptions for up to 2 years. ${ }^{19}$ That study also revealed that nearly half of all patients who were switched to another antipsychotic were likely to have been underdosed, while patients whose antipsychotic was augmented by another had frequently undergone the change before having been tested, in the opinion of the investigators, with an adequate number of alternative antipsychotic medications in monotherapy. Patients requiring a treatment switch in our study were more likely to be reported as nonadherent. This in itself is a risk factor for treatment failure, ${ }^{20,21}$ poorer clinical and functional outcomes, ${ }^{20,22,23}$ and increased risk of hospitalization. ${ }^{22,23}$ By contrast, medication intolerability was a far less common reason for treatment change. These findings appear consistent with prior research showing that the lack of efficacy of a medication, rather than its intolerability, is the most frequent cause of treatment discontinuation. ${ }^{24}$

The current findings highlight the still unmet treatment needs of patients with schizophrenia - the need for a more effective and well-tolerated medication. As potential treatments with different mechanisms of action continue to be investigated, it remains important to characterize reasons for switching versus augmentation and to develop evidencebased guidance for clinicians that defines parameters for clinicians related to switching medications versus augmentation with another agent.

Several limitations to this study bear mention. The most obvious limitation was its small sample sizes, which limited our ability to use statistical analyses to interpret the findings.
Moreover, as this was a naturalistic study, patients were not randomly assigned to treatment. As a result, we were required to rely primarily on descriptive statistics and use effect sizes to estimate the relevance of the observed patient group differences. No adjustment was made for multiplicity of observations. Moreover, while lack of efficacy was revealed to be the major driver of the decision to change patients' treatments in the case of both switching and augmentation, no information was available regarding which specific symptom or symptoms were of greatest concern to physicians during either situation. Related to this is the finding that patients characterized by both a switch and an augmentation were noted by their physicians to have demonstrated an "inadequate response." However, this may have been driven by the limited options available to choose from in this study (for example, "inadequate response," "intolerance to drug," or "upon patient's request"). If the clinicians had been provided more refined response options, the differences between the Switched and Augmented groups might have been more accurately captured (that is, the Switched group had truly inadequate responses, whereas the Augmented group merely had suboptimal responses). Another limitation is the study's inability to identify the cases in which augmentation of the current antipsychotic was actually a manifestation of an ongoing cross-titration with another antipsychotic (that is, a planned switch), which was aborted by the physician due to temporary or sustained improvement in the patient's clinical or functional status. ${ }^{1}$

\section{Conclusion}

This post-hoc and preliminary analysis suggests that clinicians tend to switch a patient's antipsychotic medication when symptoms worsen or show a lack of meaningful improvement, whereas they appear more likely to augment with another antipsychotic medication when the patient shows some improvement. However, this conclusion must be considered preliminary due to the study's limitations. Confirmation will require further research.

\section{Acknowledgment}

Appreciation is expressed to Angela Lorio, ELS, of i3, part of the inVentiv Health Company, for her expert editorial review of the manuscript.

\section{Disclosures}

This work was sponsored by Eli Lilly and Company. All authors are employees and stakeholders in Eli Lilly and Company. 


\section{References}

1. Correll CU. Antipsychotic polypharmacy, part 1: Shotgun approach or targeted cotreatment? J Clin Psychiatry. 2008;69(4):674-675.

2. Weiden PJ. Discontinuing and switching antipsychotic medications: understanding the CATIE schizophrenia trial. J Clin Psychiatry. 2007; 68(Suppl 1):S12-S19.

3. Buchanan RW, Kreyenbuhl J, Kelly DL, et al; Schizophrenia Patient Outcomes Research Team (PORT). The 2009 schizophrenia PORT psychopharmacological treatment recommendations and summary statements. Schizophr Bull. 2010;36(1):71-93.

4. Leucht S, Heres S, Kissling W, Davis JM. Evidence-based pharmacotherapy of schizophrenia. Int J Neuropsychopharmacol. 2011;14(2): 269-284.

5. Miller AL, Hall CS, Buchanan RW, et al. The Texas Medication Algorithm Project antipsychotic algorithm for schizophrenia: 2003 update. J Clin Psychiatry. 2004;65(4):500-508.

6. Barnes TR, Paton C. Antipsychotic polypharmacy in schizophrenia: benefits and risks. CNS Drugs. 2011;25(5):383-399.

7. Pandurangi AK, Dalkilic A. Polypharmacy with second-generation antipsychotics: a review of evidence. J Psychiatr Pract. 2008;14(6): 345-367.

8. Taylor D, Mir S, Mace S, et al. Co-prescribing of atypical and typical antipsychotics - prescribing sequence and documented outcome. Psychiatr Bull. 2002;26:170-172.

9. Kelin K, Lambert TJ, Brnabic AJ, et al. Treatment discontinuation and clinical outcomes in the 1-year naturalistic treatment of patients with schizophrenia at risk of treatment nonadherence. Patient Prefer Adherence. 2011;5:213-222.

10. Guy W. ECDEUAssessment Manual for Psychopharmacology, Revised. Publication ADM 76-338. Bethesda, MD: United States Department of Health, Education, and Welfare; 1976.

11. Ware J Jr, Kosinski M, Keller SD. A 12-Item Short-Form Health Survey: construction of scales and preliminary tests of reliability and validity. Med Care. 1996;34(3):220-233.

12. EuroQol Group. EuroQol - a new facility for the measurement of health-related quality of life. Health Policy. 1990;16(3):199-208.
13. Hogan TP, Awad AG, Eastwood R. A self-report scale predictive of drug compliance in schizophrenics: reliability and discriminative validity. Psychol Med. 1983;13(1):177-183.

14. Amador XF, Strauss DH, Yale SA, Flaum MM, Endicott J, Gorman JM. Assessment of insight in psychosis. Am J Psychiatry. 1993;150(6): 873-879.

15. Kaplan EL, Meier P. Nonparametric estimation from incomplete observations. J Am Stat Assoc. 1958;53(282):457-481.

16. Cohen J. Statistical Power Analysis for the Behavioral Sciences. New York, NY: Academic Press; 1969.

17. Ganguli R. Rationale and strategies for switching antipsychotics. $A m$ J Health Syst Pharm. 2002;59(22 Suppl 8):S22-S26.

18. Hugenholtz GW, Heerdink ER, Meijer WE, Stolker JJ, Egberts AC, Nolen WA. Reasons for switching between antipsychotics in daily clinical practice. Pharmacopsychiatry. 2005;38(3):122-124.

19. Tsutsumi C, Uchida H, Suzuki T, et al. The evolution of antipsychotic switch and polypharmacy in natural practice - a longitudinal perspective. Schizophr Res. 2011;130(1-3):40-46.

20. Ayuso-Gutiérrez JL, del Río Vega JM. Factors influencing relapse in the long-term course of schizophrenia. Schizophr Res. 1997;28(2-3): 199-206.

21. Lindström E, Bingefors K. Patient compliance with drug therapy in schizophrenia. Economic and clinical issues. Pharmacoeconomics. 2000;18(2):106-124.

22. Ascher-Svanum H, Faries DE, Zhu B, Ernst FR, Swartz MS, Swanson JW. Medication adherence and long-term functional outcomes in the treatment of schizophrenia in usual care. J Clin Psychiatry. 2006; 67(3):453-460.

23. Lindenmayer JP, Liu-Seifert H, Kulkarni PM, et al. Medication nonadherence and treatment outcome in patients with schizophrenia or schizoaffective disorder with suboptimal prior response. J Clin Psychiatry. 2009;70(7):990-996.

24. Lieberman JA, Stroup TS, McEvoy JP, et al; Clinical Antipsychotic Trials of Intervention Effectiveness (CATIE) Investigators. Effectiveness of antipsychotic drugs in patients with chronic schizophrenia. $N$ Engl J Med. 2005;353(12):1209-1223.
Neuropsychiatric Disease and Treatment

\section{Publish your work in this journal}

Neuropsychiatric Disease and Treatment is an international, peerreviewed journal of clinical therapeutics and pharmacology focusing on concise rapid reporting of clinical or pre-clinical studies on a range of neuropsychiatric and neurological disorders. This journal is indexed on PubMed Central, the 'PsycINFO' database and CAS.

\section{Dovepress}

The manuscript management system is completely online and includes a very quick and fair peer-review system, which is all easy to use. Visit http://www.dovepress.com/testimonials.php to read real quotes from published authors. 